\title{
UNA PROPUESTA DIDÁCTICA PARA LA CARACTERIZACIÓN DE DISPOSITIVOS Y REDES ELÉCTRICAS DE RADIOFRECUENCIA EN LA BANDA DE 100 KHZ A 500 MHZ
}

\section{A DIDACTIC PROPOSAL FOR THE CHARACTERIZATION OF RADIO FREQUENCY DEVICES AND ELECTRICAL NETWORKS AT RANGE 100 KHZ TO 500 MHZ}

\author{
R. Linares y Miranda ${ }^{1} \quad$ H. E. González-Jaimes ${ }^{2} \quad$ J. López-Bonilla $^{2}$ \\ Recibido 9 de marzo de 2010, aceptado 11 de noviembre de 2010 \\ Received: March 9, $2010 \quad$ Accepted: November 11, 2010
}

\begin{abstract}
RESUMEN
En este artículo se presenta un esquema didáctico de prueba y medición para la caracterización en el dominio de la frecuencia de componentes y circuitos lineales de radiofrecuencia (RF). El esquema se basa en la observación del comportamiento temporal manifestado por las tensiones incidentes y reflejadas. Éstas se pueden generar cuando se suministran señales sinusoidales de RF en los puertos o terminales de los dispositivos o redes eléctricas bajo estudio. El objetivo principal de este trabajo es el de proponer un método experimental alternativo que permita estudiar fenómenos que son abordados normalmente -a nivel licenciatura- en diversos programas de ingeniería eléctrica (o ramas afines). El método puede aplicarse en asignaturas donde se estudian tópicos vinculados al análisis de redes eléctricas. También en tópicos como la propagación de señales en líneas de transmisión, el diseño y operación de antenas y en los principios fundamentales de la compatibilidad electromagnética, entre otros.
\end{abstract}

Palabras clave: Enseñanza de la ingeniería, medición e instrumentación de RF, caracterización experimental de dispositivos y redes de alta frecuencia, compatibilidad electromagnética, tecnología educativa.

\section{ABSTRACT}

In this article a didactic outline of proof and measurement is presented for the characterization in frequency domain of components and linear circuits of radio frequency $(R F)$. The outline is based on the observation of the temporary behavior shown by the incident and reflected tensions. These can be generated when RF sinusoidal signals are provided at ports and terminals of electrical networks or devices under study. The main goal of this paper is to propose an alternative experimental method that allows the study of phenomena normally addressed at the undergraduate level in various programs of Electrical Engineering or related disciplines. The method can be applied to subjects where topics related to the analysis of electrical networks are covered. It can also be applied to topics such as signal propagation in transmission lines, design and operation of antennas, and the fundamental principles of electromagnetic compatibility, among others.

Keywords: Engineering teaching, RF measure and instrumentation, experimental characterization of devices and networks at high frequency, electromagnetic compatibility, educational technology.

\section{INTRODUCCIÓN}

A pesar de la importancia que el estudio del comportamiento de dispositivos y redes eléctricas lineales a excitaciones temporales de carácter sinusoidal en régimen estacionario tiene en la formación académica de todo estudiante de ingeniería eléctrica (y ramas afines) a nivel de licenciatura
[1-5], el tratamiento del mismo a escala de laboratorio en dichos programas suele en general estar limitado (llegando incluso a ser prácticamente inexistente en algunas escuelas), debido en parte a que mucha de la infraestructura experimental que se ofrece actualmente en el mercado para llevar a cabo prácticas del estudio mencionado presenta algunos inconvenientes que limitan

1 Instituto Politécnico Nacional, Escuela Superior de Ingeniería Mecánica y Eléctrica, Unidad Zacatenco, Edif. "Z” acceso Z-3, 3er piso, Lab. de Instrumentación Electrónica, Col. Lindavista, C.P. 07738, México D.F. E-mail: rlinaresy@ipn.mx

2 Instituto Politécnico Nacional, Escuela Superior de Ingeniería Mecánica y Eléctrica, Unidad Zacatenco, Edif. “Z” acceso Z-2, 3er piso, Academia de Electromagnetismo, Col. Lindavista, C.P. 07738, México, D.F. E-mail: hjaimes@ipn.mx; joseluis.lopezbonilla@gmail.com 
su uso a escala masiva, siendo principalmente el costo, disponibilidad en el mercado, necesidad de entrenamiento especializado para su operación y mantenimiento, entre otros. En la actualidad otra limitante crítica para el entendimiento de los fenómenos que se presentan en las redes eléctricas lineales es el uso de sistemas automatizados como son: medidores RLC, analizadores de impedancia, analizadores de redes, etc. [6-8].

El esquema experimental propuesto en este artículo puede considerarse esencialmente como una versión "manual" simplificada de los modernos analizadores vectoriales de redes para radiofrecuencia (RF) y microondas [9], que tiene como virtud no solo ser más económico respecto a los sistemas automatizados, sino que permite al estudiante manipular directamente los accesorios e instrumentos de medición que permiten observar el comportamiento temporal de los fenómenos de la transmisión y reflexión de señales en las líneas en transmisión, procesos que pueden utilizarse para la caracterización del comportamiento real en frecuencia de componentes pasivos $u$ otros dispositivos.

Algunos de los temas típicos de análisis redes eléctricas de dos puertos que operan en el régimen armónico de pequeña señal pueden ser fácilmente entendidos con el esquema de medición propuesto. Esto hace que los alumnos se familiaricen desde etapas tempranas de su formación de manera en la cual el análisis del comportamiento de los fenómenos antes mencionados constituye la base de algunos de los métodos de estudio teórico-experimentales más empleados en la actualidad para evaluar (tanto cualitativa como cuantitativamente) algunas de las características de operación de una gran variedad de componentes y redes eléctricas que operan en condiciones de "alta frecuencia" [1-3].

\section{METODOLOGÍA}

\section{a) Principio de operación del esquema experimental didáctico propuesto}

Por razones de carácter fundamentalmente experimental $[1,2,4]$, se conoce que de todos los parámetros existentes para caracterizar la respuesta "a pequeña señal" de componentes y circuitos (tanto pasivos como activos) de "N" puertos con excitación sinusoidal de alta frecuencia en régimen estacionario, los parámetros " $\mathrm{S}$ " (o de dispersión) resultan ser actualmente -a diferencia de los parámetros de impedancia ' $Z$ ', de admitancia " $Y$ ", híbridos " $h$ " y otros- los más utilizados en la práctica para llevar a cabo dicha labor.
Los parámetros de dispersión (a diferencia de las otras clases de parámetros mencionados) se basan en el hecho de que al momento de conectar cualquier clase de generador de RF a todo componente o red eléctrica de carácter lineal en alguno de sus puertos, parte de la tensión, corriente y potencia que se suministra será reflejada hacia dicho generador, debido a que no se cumple con el teorema de la máxima transferencia de potencia, lo cual significa que entre el generador y la red eléctrica en cuestión existe un desacoplamiento de impedancia, es decir $[1,10]$ :

$$
Z_{\text {gen }} \neq Z_{i i}^{*}
$$

donde $Z_{g e n}$ es la impedancia interna (i.e., impedancia del equivalente Thévenin) del generador que excita al circuito o dispositivo y $Z_{i i}$ es la impedancia del "i-ésimo" puerto asociado al componente, arreglo o circuito, que corresponde a la "impedancia de carga", por lo que también se representa por $Z_{c a r}$.

Si se cumple con el teorema de la máxima transferencia de energía (es decir: $Z_{g e n}=Z_{i i}^{*}$ ), entonces no se presenta tensión, corriente ni potencia reflejadas. En casos como este se dice que se tiene acoplamiento de impedancia entre el generador y la carga. En cambio, cuando el teorema de máxima transferencia de potencia no se cumple -tal y como lo establece la condición (1) -, entonces la tensión reflejada de la carga al generador $V_{r e f}(t)$ se hará presente entre dichos elementos, teniendo dicha tensión una amplitud y fase cuyos valores están vinculados estrechamente con la amplitud y fase correspondientes a la tensión incidente $V_{\text {inc }}(t)$ que el generador suministra al puerto o "carga". Una forma ampliamente usada de expresar en forma analítica la interdependencia entre las características de amplitud $\mathrm{y}$ fase de dichas tensiones es la siguiente:

$$
\frac{\tilde{V}_{r e f}}{\tilde{V}_{i n c}}=\Gamma=\frac{Z_{c a r}-Z_{g e n}}{Z_{c a r}+Z_{g e n}}
$$

donde $\tilde{V}_{r e f}$ y $\tilde{V}_{i n c}$ son las representaciones fasoriales de las tensiones reflejada e incidente en la carga; siendo " $\Gamma$ " el coeficiente de reflexión para tensión que se presenta en el puerto de entrada de la red o carga del generador. Dado que todas las cantidades aludidas en la expresión anterior son -en general- complejas, esto quiere decir que " $\Gamma$ " podría ser reescrita así:

$$
\Gamma=|\Gamma| \angle \theta_{\Gamma}
$$


Expresión esta última de la cual es posible demostrar que se pueden definir -de acuerdo a (1) y (2)- las siguientes cantidades:

$$
\begin{gathered}
|\Gamma|=\frac{\left|\tilde{V}_{r e f}\right|}{\left|\tilde{V}_{i n c}\right|} \\
\theta_{\Gamma}=\theta_{r e f}-\theta_{i n c}=\Delta \theta
\end{gathered}
$$

Siendo $\left|\tilde{V}_{\text {ref }}\right|$ y $\left|\tilde{V}_{\text {inc }}\right|$ las amplitudes máximas de las tensiones reflejada e incidente (respectivamente) que se presentan en la carga; en tanto que $\Delta \theta$ representa la proporción y clase de desfasamiento que existe entre dichas tensiones.

Si el valor de una de las impedancias que están vinculadas en la determinación de $\Gamma$ es conocido (normalmente es la $Z_{g e n}$ ), entonces puede constatarse que:

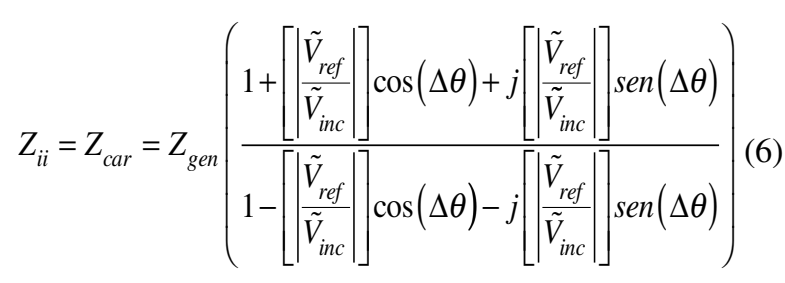

Otro parámetro que se relaciona con el acoplamiento y desacoplamiento entre la impedancia interna del generador y la impedancia de entrada de la red o la impedancia de carga es la razón de onda estacionaria de tensión (ROEV), que también se conoce como VSWR por sus siglas en inglés. Este parámetro es un factor de mérito en el acoplamiento de redes y es esencial para el desarrollo de antenas. El ROEV se produce en un puerto o carga de la red eléctrica y puede calcularse en estos casos a partir del coeficiente de reflexión dado por (2). La expresión para el cálculo de la ROEV es la siguiente:

$$
\operatorname{ROEV}=\frac{1+\left(\left|\tilde{V}_{\text {ref }}\right| /\left|\tilde{V}_{\text {inc }}\right|\right)}{1-\left(\left|\tilde{V}_{\text {ref }}\right| /\left|\tilde{V}_{\text {inc }}\right|\right)}
$$

La importancia de estas dos últimas expresiones radica en el hecho de que pueden calcularse o estimarse los valores tanto de la $Z_{c a r}$ conectada como de su correspondiente ROEV usando en las mismas los valores de las cantidades $\left|\tilde{V}_{\text {ref }}\right|,\left|\tilde{V}_{\text {inc }}\right|$ y $\Delta \theta$, las cuales -a su vez-pueden medirse en forma directa con poca dificultad empleando el método experimental cuya estructura y principio de operación se presentan y discuten a continuación.

\section{b) Descripción del esquema didáctico de medición propuesto en su modalidad de "reflexión"}

Con base en esta última idea, se encontró que una forma relativamente simple (y a la vez muy ilustrativa) de observar el comportamiento de cada una de las tensiones descritas en situaciones como la ya referida es mediante el uso de un arreglo de prueba cuyo elemento central es un dispositivo conocido como "unidad para pruebas de transmisión/reflexión (T/R)" -T/R test set- de la marca "Hewlett Packard" (modelo 41952A), el cual forma parte de instrumentos de medición especializados en la caracterización dinámica de componentes y redes a "pequeña señal" (como es el caso de los analizadores vectoriales de redes) [3], el cual opera en el intervalo de $100 \mathrm{kHz}$ a $500 \mathrm{MHz}$. Dicho conjunto es capaz de poder "separar" de manera simultánea las tensiones incidente, reflejadas $\mathrm{y}$ transmitida que se producen al momento de conectar a su puerto "de salida o de prueba" toda clase de elementos o red cuya impedancia en ese par de terminales pudiese llegar a ser distinta a la propia de este conjunto (la cual es de 50 Ohms) con un alto grado de "confiabilidad" desde el punto de vista metrológico [10]. Tomando en cuenta esto último, el esquema de medición propuesto en este trabajo es el que se muestra en la Figura 1.

Con respecto a este montaje de prueba, cabe mencionar que el osciloscopio a usar en casos como este puede ser tanto analógico como digital, siempre y cuando el mismo tenga un ancho de banda que cubra (al menos) una parte importante del que posea la unidad de T/R empleada. Pese a ello se aconseja usar un osciloscopio digital de doble trazo con despliegue a color y capacidad de ajuste de su impedancia de entrada (en cada uno de sus canales) a 50 Ohms, para lograr con ello no solo simplificar las tareas de visualización e interpretación de las formas de onda obtenidas, sino también para facilitar las tareas de acoplamiento de impedancia que es deseable existan entre los canales del mismo con los puertos correspondientes del mencionado conjunto. 


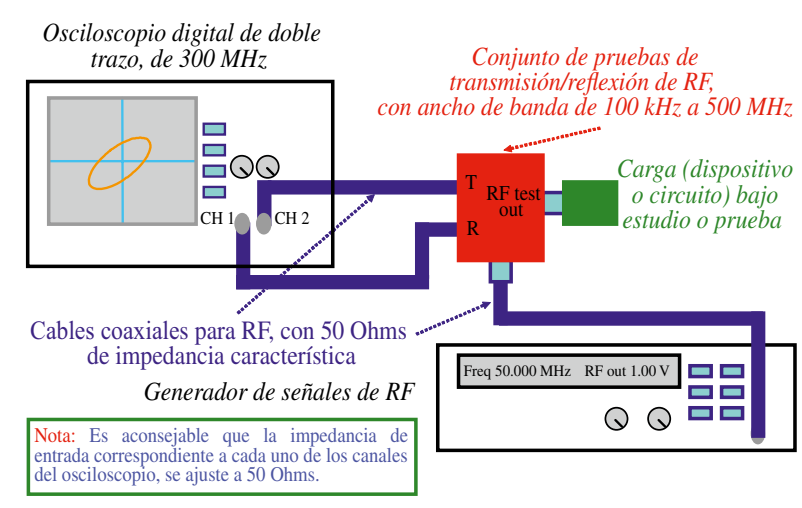

Figura 1. Representación pictórica de la propuesta didáctica para determinar el coeficiente de reflexión para voltaje (ROEV) y la impedancia en componentes de RF, en la banda de $100 \mathrm{kHz}$ a $500 \mathrm{MHz}$.

Una vez montado el arreglo mostrado en la Figura 1, la forma en que se debe proceder para medir cada una de las cantidades descritas por las ecuaciones (6) y (7) haciendo uso del mismo es la siguiente:

1) Conectar el dispositivo bajo prueba (DBP) o el circuito bajo prueba (CBP) a la terminal "RF TEST OUT" de la Unidad de Transmisión/Reflexión (T/R).

2) Suministrar a la terminal "RF INPUT" de la citada Unidad la señal sinusoidal a usar en la prueba, con la amplitud y frecuencia deseadas.

3) Medir en el osciloscopio tanto la amplitud máxima de cada una de las tensiones que se obtienen en los puertos marcados como: "T" (reflejada) y "R" (incidente) de la Unidad de $T / R$, así como el valor correspondiente al desfasamiento mutuo existente entre ellas, tomando como referente la señal obtenida del puerto " $R$ ". El puerto " $T$ " (en este caso) se observa en el canal 1 del osciloscopio y el puerto " $R$ " en el canal 2 del mismo, luego de haber ajustado las escalas de deflexión vertical correspondientes a los canales ya mencionados.

4) Con los valores de $\left|\tilde{V}_{\text {ref }}\right|,\left|\tilde{V}_{\text {inc }}\right|$ y $\Delta \theta$ obtenidos de la medición especificada en el punto anterior y utilizando las expresiones (3)-(7), se procede a calcular los valores de $\Gamma, Z_{c a r}$ y ROEV a la frecuencia que posee la señal de prueba suministrada por el generador, tomando en cuenta que el valor de la $Z_{g e n}$ en cada una de estas pruebas es de $50+0 j$ Ohms.

Nota. Para determinar el signo que posee el coeficiente de reflexión a estimar (importante para definir el carácter reactivo de la impedancia en cuestión) se recomienda al lector consultar la referencia [11], para familiarizarse con el procedimiento que debe seguirse para determinar el grado de "atraso" o "adelanto" que posee la tensión reflejada obtenida con respecto a la incidente suministrada a través de la Unidad de T/R para RF antes descrita.

\section{RESULTADOS}

Para determinar cuál podría ser el grado de confiabilidad específico de este método didáctico experimental en la cuantificación de cada una de las cantidades mencionadas, se procedió primero a determinar los valores del coeficiente de reflexión en la banda de frecuencias antes mencionada, producido por un arreglo formado por un resistor convencional de película de carbón, conectado en serie a un capacitor cerámico de baja tensión, siendo 15 Ohms y 330 picofarads los valores nominales respectivos de estos componentes. Dicho arreglo se montó sobre un conector coaxial para RF del tipo "N" hembra para chasis de propósito general, con el fin de facilitar la conexión de sus terminales con el "puerto de prueba" de la Unidad de T/R utilizado en este arreglo. Acto seguido se aplicó una señal de RF cuyo nivel se fijó a $+13 \mathrm{dBm}$, procediendo luego a modificar la frecuencia de la misma en valores comprendidos desde $100 \mathrm{kHz}$ hasta $500 \mathrm{MHz}$.

Para tener una idea más precisa del grado de exactitud alcanzado por la estimación experimental de los valores correspondientes al coeficiente de reflexión propio de este arreglo (obtenidos luego de aplicar el método antes descrito), se efectuó posteriormente la medición de este parámetro en un Analizador de Impedancias para RF de la marca "Hewlett Packard" (modelo 4191A), haciendo uso de la opción de corrección de longitud eléctrica que este instrumento tiene incorporada, con el propósito de compensar los errores de medición que se producen por los efectos "de retardo" de señal introducidos al emplear distintas clases de conectores y adaptadores para RF entre el puerto de salida y el arreglo RC bajo prueba [12]. En el caso de esta prueba, dicha corrección se realizó obteniendo primero el "retardo" introducido por el adaptador y el conector coaxial empleados para suministrar la señal de prueba al arreglo, sin que este último estuviese colocado sobre el conector "N" ya descrito, para luego "descontar" o compensar dicho retardo adicional del obtenido al momento de medir con el osciloscopio el desfasamiento existente entre los voltajes incidente y reflejado obtenidos después de soldar el arreglo al mencionado conector.

En las Figuras $2 \mathrm{a}$ y $2 \mathrm{~b}$ se muestran (respectivamente) los valores correspondientes a la magnitud y la fase del coeficiente generado por el arreglo RC ya descrito en el intervalo de frecuencias indicado anteriormente, luego 
de comparar las características que poseían los voltajes incidente y reflejado producidos por el mismo (de acuerdo al procedimiento de análisis propuesto en este trabajo), junto con los determinados experimentalmente con la ayuda del Analizador de Impedancias HP4191A, siguiendo en este último caso el procedimiento descrito en el párrafo anterior.

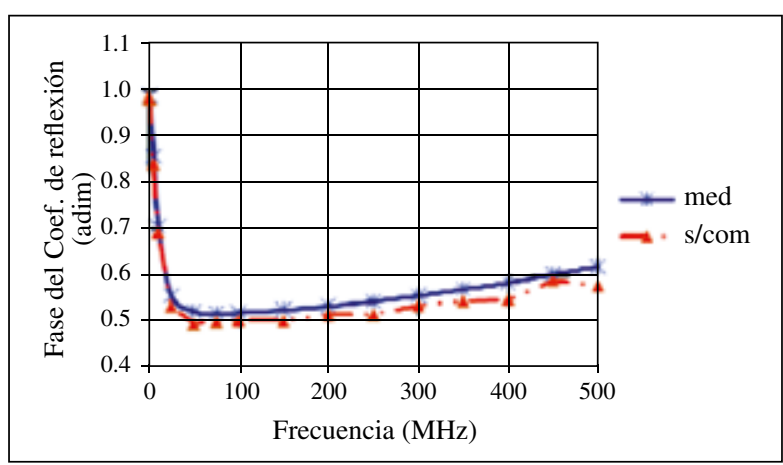

Figura 2a. Magnitud del coeficiente de reflexión del arreglo RC descrito en el texto, según: "med", la medición efectuada con un analizador de impedancias, "s/com" con el método propuesto, usando los valores "no corregidos" del ángulo de fase entre las tensiones incidente y reflejada.

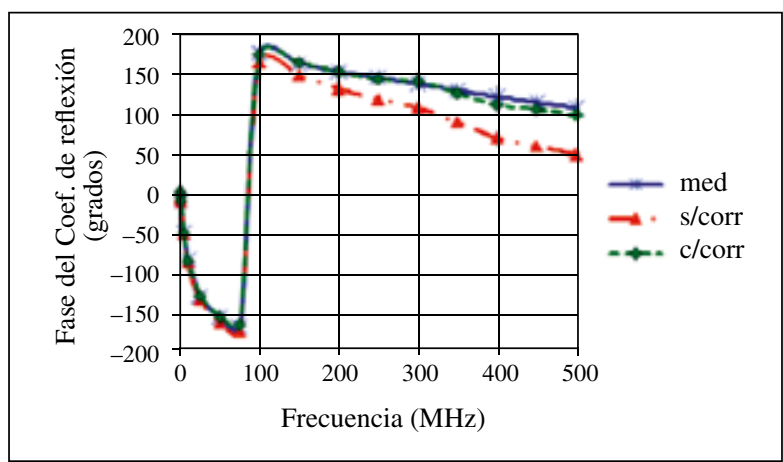

Figura 2b. Ángulo de fase correspondiente al coeficiente de reflexión del arreglo $\mathrm{RC}$ (incluyendo también los resultados "c/corr", obtenidos al considerar la "corrección" del desfasamiento que se presenta entre las tensiones incidente y reflejada).

Obtenido el coeficiente de reflexión producido por el arreglo RC bajo prueba en cada una de las frecuencias especificadas anteriormente, la impedancia equivalente de dicho arreglo se calcula por medio de la ecuación (6), considerando que la $Z_{\text {gen }}$ es igual a $50+0 j$ ohms. Los valores obtenidos tanto para la parte resistiva como la parte reactiva de la impedancia antes mencionada -luego de aplicar los procesos de estimación antes descritos-aparecen representados en cada una de las gráficas que componen las Figuras 3a y 3b que se muestran a continuación:

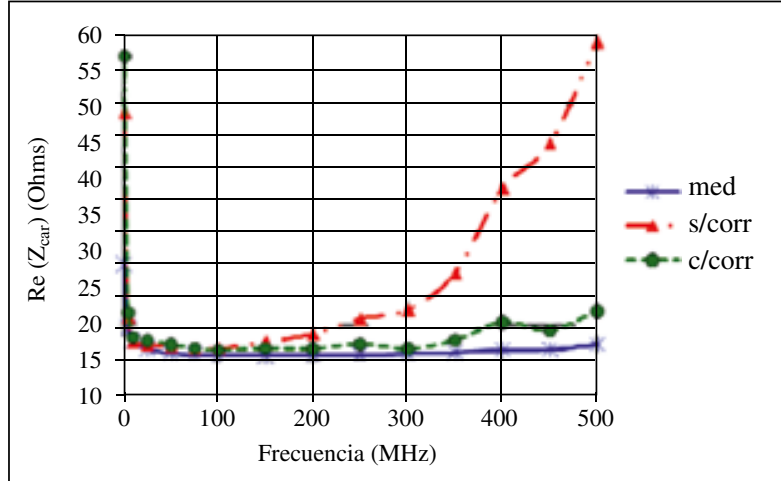

Figura 3a. Comportamiento de la parte resistiva de la impedancia del arreglo RC; según: "med", la medición efectuada en un analizador de impedancias, "s/corr" usando los valores "no corregidos" del coeficiente de reflexión y "c/corr", tomando en cuenta los valores ya "corregidos" de dicha cantidad.

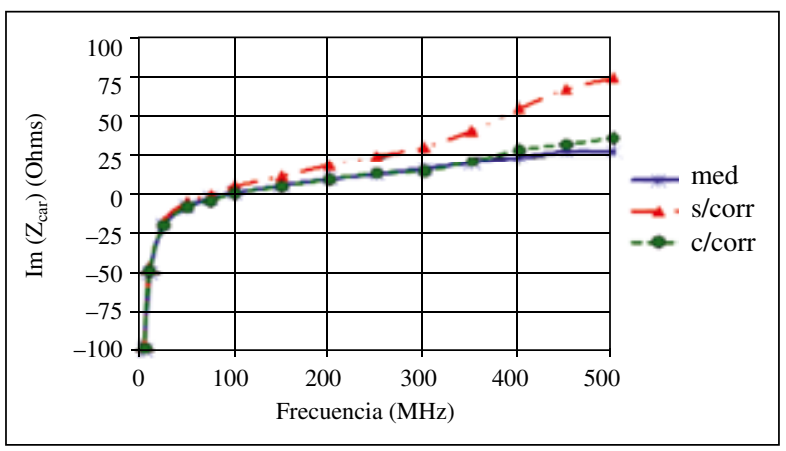

Figura 3b. Comportamiento de la parte reactiva de la impedancia del arreglo RC; de conformidad a los resultados obtenidos en cada uno de los procedimientos experimentales descritos en este artículo.

La otra prueba seleccionada para validar el grado de certidumbre que puede brindar el montaje experimental descrito consistió en evaluar el comportamiento en frecuencia de la razón de onda estacionaria propia de una antena comercial de banda ancha, empleada en pruebas de Compatibilidad Electromagnética (EMC por sus siglas en inglés), para evaluar toda clase de interferencias electromagnéticas radiadas en la banda de VHF.

El dispositivo seleccionado para llevar a cabo esta prueba fue la antena bicónica modelo 3110B de la empresa ETSLindgren. La disponibilidad de la misma en el Laboratorio de Compatibilidad Electromagnética de la SEPI-ESIME Zacatenco del Instituto Politécnico Nacional (así como el hecho de que su ancho de banda se ajustara muy bien al intervalo de frecuencias manejado en la propuesta experimental tratada en este trabajo) fueron las razones 
principales que motivaron a seleccionar esta antena para efectuar dicha prueba. Una vez colocada en el interior de la cámara anecoica del mencionado Laboratorio, se suministraron a la misma señales cuya frecuencia iba desde los $30 \mathrm{MHz}$ hasta los $300 \mathrm{MHz}$, en pasos de 30 MHz. Debido a que el parámetro a evaluar en este caso era la ROEV generada por la antena en sus terminales de conexión, se observó que no era necesario en este caso aplicar a las mediciones de tensión incidente y reflejada llevadas a cabo con la unidad de T/R la corrección de fase descrita anteriormente. Con las magnitudes de estas últimas y aplicando lo descrito en la expresión (7), se calculó el valor de la ROEV correspondiente a este dispositivo en dichas frecuencias, para luego comparar todos y cada uno de los resultados obtenidos en dicho ensayo con lo reportado por el fabricante de esta antena. El resultado de todo lo descrito en este párrafo se muestra en las gráficas que componen las Figuras $4 \mathrm{a}$ y $4 \mathrm{~b}$, respectivamente.

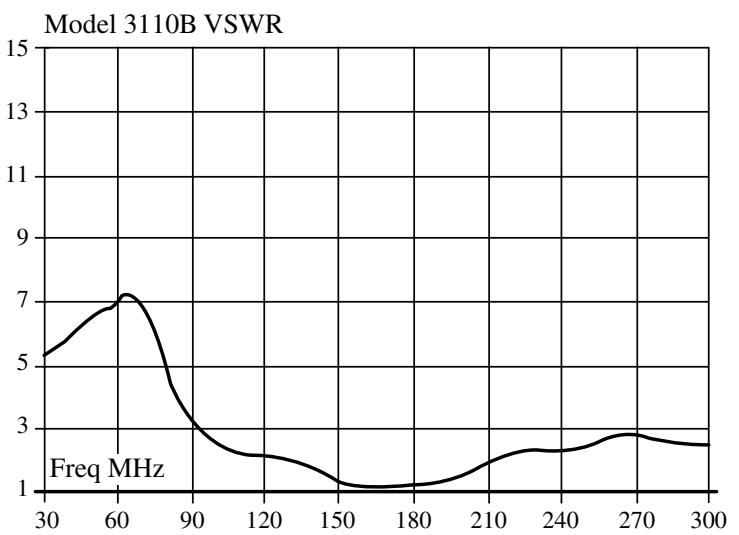

Figura 4a. Comportamiento de la ROE manifestado por la antena bicónica para EMC 3110B, según la información proporcionada por el fabricante.

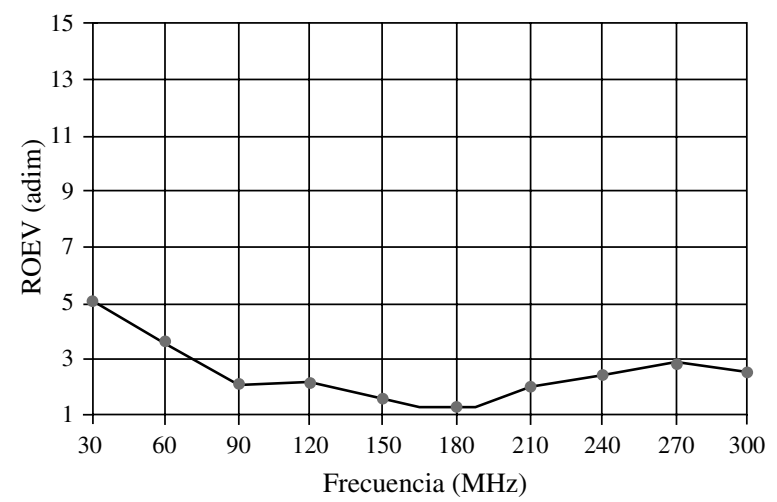

Figura 4b. Comportamiento de la ROE manifestado por la antena bicónica para EMC 3110B, de acuerdo al método de evaluación experimental propuesto a lo largo de este trabajo.

\section{DISCUSIÓN}

En el caso de la primera de las pruebas reportadas en este artículo, Figura 2b, se puede apreciar que el comportamiento manifestado por el ángulo del coeficiente de reflexión para tensión " $\Gamma$ " del arreglo RC bajo prueba en el intervalo de frecuencias usado en dicho experimento difiere de manera importante del teórico que se esperaría obtener -en primera instancia- del mismo, ya que el ángulo $\theta_{\Gamma}$ propio de este tipo de carga se comporta como función monotónica decreciente de la frecuencia (según el comportamiento "ideal" en frecuencia esperado de cada uno de los componentes de este arreglo); siendo el límite asintótico correspondiente igual a $-180^{\circ}$. Como puede verse claramente en la gráfica ya mencionada, esto solo ocurre así hasta los $80 \mathrm{MHz}$, ya que a partir de dicha frecuencia el valor de este ángulo cambia abruptamente a cerca de $+180^{\circ}$, para luego ir disminuyendo de manera progresiva si la frecuencia de la señal de RF aplicada supera el umbral ya mencionado sin que el mismo ( $\sin$ embargo) llegue a ser otra vez negativo.

Una explicación que se puede dar a este fenómeno radica en el hecho de que resulta probable el que a partir de esa frecuencia, se hacen significativos los efectos de segundo orden propios de los dispositivos empleados en la fabricación de la carga bajo estudio [11] (en particular el carácter inductivo propio de cada una de las terminales de conexión del resistor y el capacitor, aunado al del "poste" metálico que se usó para conectar un extremo del arreglo al cuerpo del conector en el cual se montó el circuito RC bajo prueba).

Otro aspecto que cabe resaltar es la importancia que pueden llegar a tener -en un momento dado- dichos fenómenos o efectos de alto orden en la determinación exacta de valores como el coeficiente de reflexión y la impedancia de dispositivos o redes a frecuencias cada vez más altas. Tal y como se puede apreciar en las gráficas propias de la Figura 3 existe una mejora significativa en el cálculo de esta cantidad a frecuencias por arriba (en este caso) a los $300 \mathrm{MHz}$ que se puede lograr aplicando la metodología experimental presentada en este trabajo, si se considera a los adaptadores y conectores coaxiales para RF empleados en la interconexión de la unidad de $\mathrm{T} / \mathrm{R}$ con el CBP (en primera aproximación) como una red eléctrica de parámetros distribuidos (léase una línea de transmisión ideal o sin pérdidas), que actúa en situaciones como esta en forma de una red eléctrica de "retardo" no disipativa, debido esencialmente a que su longitud eléctrica equivalente comienza a ser ya comparable con la longitud de onda asociada a la señal de RF que se transfiere (en forma de ondas electromagnéticas) a través de los accesorios antes mencionados [12]. 


\section{CONCLUSIONES}

En este trabajo se presentó un esquema experimental de naturaleza didáctica, orientado a la caracterización o estudio del comportamiento en frecuencia de algunos componentes y circuitos pasivos de RF en la banda que va de $100 \mathrm{kHz}$ a $500 \mathrm{MHz}$, vía el cálculo o estimación del valor de la impedancia asociada a los elementos bajo prueba, a través del análisis de cómo se comporta el coeficiente de reflexión para tensión que se genera por parte de los elementos bajo prueba sobre ciertos dispositivos de impedancia conocida, mediante la observación de las características temporales de las tensiones incidente y reflejada que se producen en el punto de interconexión de los mencionados dispositivos, a través de un osciloscopio digital de propósito general de banda ancha.

Desde el punto de vista didáctico, este montaje de medición y prueba posee ciertos atributos que pueden ser viables y atractivos en su empleo para prácticas de laboratorio de ciertas asignaturas (a nivel licenciatura), relacionadas con el estudio del comportamiento dinámico de dispositivos y redes en función de la frecuencia. Los puntos importantes de conclusión son:

a) Hace uso (en su gran mayoría) de instrumentos, accesorios y componentes que están presentes en la gran mayoría de los laboratorios universitarios de ingeniería electrónica.

b) Algunos de los elementos de carácter semiespecializado requeridos por este sistema (la unidad de T/R en particular) son de costo relativamente bajo y no resulta complicado adquirir dicho componente o accesorio en el mercado.

c) Hace más "comprensible" al estudiante el significado o la interpretación que puede darse a conceptos tales como el coeficiente de reflexión y la razón de onda estacionaria (por ejemplo), no solo porque "ve" (literalmente hablando) la forma en la cual el primero de ellos se produce en ciertas circunstancias, sino también porque el mismo "manipula" directamente lo observado para llegar a obtener el valor de cada una de dichas cantidades, lo cual le puede ayudar a encontrar de manera más simple la relación causa-efecto entre lo descrito matemáticamente con lo que ocurre "a nivel físico".

d) Con algunas modificaciones, este sistema puede desarrollarse a futuro para introducir al estudiante en los principios que sustentan los métodos de caracterización de algunos parámetros básicos de redes activas y pasivas de dos puertos, vía el estudio experimental detallado de los fenómenos de reflexión y transmisión de señales de RF que se pueden presentar en los mismos, teniendo para ello una instrumentación adicional o de apoyo adecuada (en particular, un osciloscopio analógico o digital de cuatro canales).

\section{REFERENCIAS}

[1] D.M. Pozar. "Microwave engineering". John Wiley and Sons, Inc. Second edition. New York, USA. 1997.

[2] Agilent AN 1287-1. "Understanding the Fundamental Principles of Vector Network Analysis". Product specifications and descriptions in this document subject to change without notice. Copyright 1997, 2000 Agilent Technologies Printed in U.S.A. 8/00 5965-7707E. 2000. URL: http://cp.literature.agilent. com/ litweb/pdf/5965-7707E.pdf. Date of visit: October 2, 2010.

[3] D.A. Bell. "Electronic Instrumentation and Measurements". Second edition. Oxford University Press. Toronto, Canada. 2007.

[4] M. Zennaro and C. Fonda. "Radio Laboratory Handbook". School on Digital Radio Communications for Research and Training in Developing Countries. UNESCO. Vol. 1. ISBN 92-95003-24-1. February, 2004.

[5] "Ondas Electromagnéticas Guiadas". Programa de la Escuela Superior de Ingeniería Mecánica y Eléctrica del Instituto Politécnico Nacional de México. México. 2006.

[6] Agilent. "Impedance Measurement Handbook. A guide to measurement technology and techniques Four Edition Agilent Technologies, Inc. 2000-2009. Printed in USA. June 17, 2009. Printed in USA. June 17, 2009. URL http://cp.literature.agilent.com/ litweb/pdf/5950-3000.pdf. Date of visit: October 25, 2010.

[7] Brooke Clarke. N6GCE. "Impedance Measurements". URL: http://www.prc68.com/I/Z.shtml. Date of visit: October 25, 2010.

[8] Olaf Ostwald. "Time Domain Measurements using Vector Network Analyzer ZVR". Rohde \& Schwarz. Application Note 1EZ44_OE. Subject to change. May 19,1998. URL: http://www2.rohde-schwarz. com/file/1ez44_Oe.pdf

[9] Anritsu. "Arbitrary Impedance". Scorpion May. Rev: B 11410-00284. Data subject to change without notice. 2002. URL: http://www.us. anritsu. com/downloads/files/11410-00284B.pdf. Date of visit: October 25, 2010.

[10] D.K. Paul and P. Gardner. "Maximum Power Transfer Theorem: A Simplified Approach". Int. J. Elect. Enging. Educ. Vol. 35, pp. 271-273. 1998.

[11] J. Getty. "Sinusoidal Steady State", pp. 2-3. Date of visit: September 30, 2009. URL: www. educatorscorner.tm.agilent.com

[12] C.R. Paul. "Introduction to Electromagnetic Compatibility". John Wiley and Sons, Inc. Chap. 6. New York, USA. 1992. 